\title{
On the constrained structure of duality symmetric Maxwell theory
}

\author{
R. Banerjeet \\ S.N. Bose National Centre for Basic Sciences \\ Block- JD, Sector III, Salt Lake City \\ Calcutta- 700,091; India
}

\begin{abstract}
The constrained structure of the duality invariant form of Maxwell theory is considered in the Hamiltonian formulation of Dirac as well as from the symplectic viewpoint. Compared to the former the latter approach is found to be more economical and elegant. Distinctions from the constrained analysis of the usual Maxwell theory are pointed out and their implications are also discussed.
\end{abstract}

\footnotetext{
${ }^{1}$ e-mail: rabin@boson.bose.res.in
} 


\section{Introduction}

The relevance of duality symmetry in either field or string theories has been reviewed [1] in various contexts. The duality invariant Lagrangeans obtained in different cases, though equivalent to their parent Lagrangeans, present features which are distinctly unique. While most investigations [1, 2, 3, [4 are directed towards undersatnding the nature of duality symmetry in such Lagrangeans, a detailed constrained analysis is lacking. Some sporadic computations [5] are available but these are incidental and are merely used for getting other results.

In this paper a detailed constrained analysis of the duality invariant form of Maxwell theory is done both in the Hamiltonian formalism of Dirac and in the symplectic approach, which is a Lagrangean formulation. We find that the constrained structure is quite distinct from the conventional Maxwell theory. Moreover, the results are obtained in a more clean and brief fashion in the symplectic approach as compared with the Dirac approach.

In section 2, the Dirac analysis is performed. The theory presents a mixed system containing both first and second class constraints. The constraints

in the second class set are not independent thereby providing an example of a reducible system. By introducing auxiliary variables [6] this reducibility is taken into account and the second class constraints are eliminated by computing the relevant Dirac brackets. Interestingly, it is observed that the knowledge of these brackets is sufficient to obtain the complete algebra among the gauge invariant variables and explicit reference to the first class constraints or any gauge fixing is avoided. The results are found to agree with the standard analysis [5] where the complete constraint sector including the Coulomb gauge was taken. We also show how to implement the axial gauge condition in the present context. The independent canonical pairs are easily identifiable in this gauge.

The symplectic treatment of the model is carried out in section 3. Once again there are striking differences from the usual constraint analysis of the Maxwell theory. It is found that the zero modes of the non-invertible symplectic two form do not lead to any new condition. This signals the presence of a gauge theory. However, none of the constraints- either first or second class- found in the Dirac scheme are obtained here. Effectively, therefore, the model simulates the features of an unconstrained theory. The noninvertibil- 
ity of the symplectic two form is due to the fact that the duality invariant Lagrangean is expressed solely in terms of the transverse components of the gauge potentials, quite in contrast to the usual Maxwell Lagrangean. Incorporating this transversality in the Lagrangean, either by means of a Lagrange multiplier or directly, the symplectic two form becomes invertible. The brackets are then easily read-off from the inverse. Since constraints do not emerge at any stage of the computations, the issue of gauge fixing is completely bypassed. Nevertheless, we explicitly show how the different gauge fixed results in the Dirac approach are reproduced in the symplectic formalism.

Some concluding remarks have been made in section 4 .

\section{Dirac Analysis}

In this section a detailed Dirac analysis of the duality symmetric electromagnetic Lagrangean will be performed. In terms of the pair of electric and magnetic fields this Lagrangean is given by the familiar expression [3, 4, 5],

$$
\mathcal{L}=\frac{1}{2}\left(B_{i}^{a} \epsilon_{a b} E_{i}^{b}-B_{i}^{a} B_{i}^{a}\right)
$$

where,

$$
\begin{gathered}
E_{i}=-F_{0 i}=-\partial_{0} A_{i}+\partial_{i} A_{0} \\
B_{i}=\epsilon_{i j k} \partial_{j} A_{k}
\end{gathered}
$$

and $a=1,2$ is an internal index charactersing the pair of fields while $\epsilon_{12}=$ $-\epsilon_{21}=1$. Expressed in terms of the potentials the Lagrangean, modulo a divergence term, simplifies to,

$$
\mathcal{L}=\frac{1}{2} \epsilon^{j k i} \partial_{j} A_{k}{ }^{a} \epsilon_{a b} \partial_{0} A^{b}{ }_{i}-\frac{1}{4} F^{a, j k} F_{j k}^{a}
$$

The above Lagrangean will be the starting point of the Dirac constrained analysis. Note that the time component of the potentials are absent so that we may, without any loss of generality, do away completely with the canonical set $\left(A_{0}, \pi^{0}\right)$. In that case the primary constraints of the system are given by,

$$
\Omega_{i}^{a}=\pi_{i}^{a}+\frac{1}{2} \epsilon_{a b} \epsilon_{i j k} \partial_{j} A_{k}^{b} \approx 0
$$


It can be checked that no further constraints are generated by demanding the time conservation of (5). Furthermore, the above set is a mixture of first and second class constraints. These may be easily separated by decomposing (5) into its transverse and longitudinal components [5]. Then the first class constraints are given by,

$$
\partial_{i} \Omega_{i}^{a}=\partial_{i} \pi_{i}^{a} \approx 0
$$

while the second class ones are,

$$
\Omega_{T, i}^{a}=\pi_{T, i}^{a}+\frac{1}{2} \epsilon_{a b} \epsilon_{i j k} \partial_{j} A_{T, k}^{b} \approx 0
$$

In order to compute the Dirac brackets the usual approach is to fix a gauge corresponding to the first class constraints (6). The Coulomb gauge,

$$
\partial_{i} A_{i}^{a} \approx 0
$$

is a popular choice. Now the complete constraint sector is given by (6), (7) and (8). Since the constraint structure is rather involved, an elaborate calculation is now necessary to explicitly get the Dirac brackets. [? The results, which are given in terms of the transverse components since these are the gauge invariant variables, are quoted from the literature [5],

$$
\begin{gathered}
\left\{A_{T, i}^{a}(x), A_{T, j}^{b}(y)\right\}^{*}=\epsilon_{a b} \epsilon_{i j k} \frac{\partial_{k}}{\nabla^{2}} \delta(x-y) \\
\left\{A_{T, i}^{a}(x), \pi_{T, j}^{b}(y)\right\}^{*}=\frac{1}{2} \delta_{a b}\left(g_{i j}+\frac{\partial_{i} \partial_{j}}{\nabla^{2}}\right) \delta(x-y) \\
\left\{\pi_{T, i}^{a}(x), \pi_{T, j}^{b}(y)\right\}^{*}=-\frac{1}{4} \epsilon_{a b} \epsilon_{i j k} \partial_{k} \delta(x-y)
\end{gathered}
$$

where,

$$
A_{T, i}^{a}(x)=\left(\delta_{i j}-\frac{\partial_{i} \partial_{j}}{\nabla^{2}}\right) A_{j}^{a}(x)
$$

All the constraints are now strongly implemented.

We now discuss an alternative formulation of the problem which highlights certain distinctive features that are otherwise hidden. Let us begin with the second class set (7). Confined to this set, the Poisson bracket matrix is given by,

$$
\left\{\Omega_{T, i}^{a}(x), \Omega_{T, j}^{b}(y)\right\}_{P B}=\epsilon_{a b} \epsilon_{i j k} \partial_{k} \delta(x-y)
$$

\footnotetext{
${ }^{2}$ These will be denoted by a star
} 
The right hand side has no inverse which, at first sight, would seem bit surprising since we are considering only the second class sector. The reason for this anomalous behaviour is that the second class constraints are reducible because,

$$
\partial_{i} \Omega_{T, i}^{a}=0
$$

so that all $\Omega_{T, i}^{a}$ are not independent. The standard way is to isolate the independent subset of constraints and proceed with the computations. Besides being messy, such an approach is generally not recommended since certain symmetries of the original problem may be lost. A particularly elegant way is to suitably modify the constraints by introducing an additional pair of canonical variables [6],

$$
\tilde{\Omega}_{T, i}^{a}(x)=\pi_{T, i}^{a}+\frac{1}{2} \epsilon_{a b} \epsilon_{i j k} \partial_{j} A_{k, T}^{b}+\partial_{i} \phi^{a} \approx 0
$$

where,

$$
\left\{\phi^{a}(x), \phi^{b}(y)\right\}=\frac{\epsilon_{a b}}{m} \delta(x-y)
$$

and a mass scale has been introduced for dimensional reasons. It is easy to see that $\partial_{i} \tilde{\Omega}_{T, i}^{a} \approx 0$ leads to $\phi^{a} \approx 0$ so that the modified constraint is weakly equivalent to the original one. Moreover, by this modification, the reducibility in the initial constraint has been removed. We can now directly work with the new constraint. A similar strategy was used earlier to analyse in details the reducibility in the constrained structure of $p$ - form gauge fields [6]. The Poisson bracket matrix among the new constraints is now given by,

$$
C^{a b}{ }_{i j}=\left\{\tilde{\Omega}_{T, i}^{a}(x), \tilde{\Omega}_{T, j}^{b}(y)\right\}=\epsilon_{a b}\left[\epsilon_{i j k} \partial_{k}-\frac{1}{m} \partial_{i} \partial_{j}\right] \delta(x-y) 3
$$

which has the following inverse,

$$
C^{a b}{ }_{, i j}^{-1}=-\epsilon_{i j k} \frac{\partial_{k}}{\nabla^{2}}-m \frac{\partial_{i} \partial_{j}}{\left(\nabla^{2}\right)^{2}}
$$

It is now straightforward to calculate the Dirac brackets among the basic variables. The result is,

$$
\left\{A_{i}^{a}(x), A_{j}^{b}(y)\right\}^{*}=\epsilon_{a b} \epsilon_{i j k} \frac{\partial_{k}}{\nabla^{\mathbf{2}}} \delta(x-y)
$$


It is simple to prove that the complete algebra (9, 10, 11) is reproduced from the above equation and using the strong implementation of the second class constraints.

It is worthwhile to note that by properly accounting for the second class sector only, the complete Dirac algebra among the physical variables has been obtained. Explicit reference to either the first class constraint or any gauge fixing has been avoided.

In usual electrodynamics the axial gauge is sometimes used since it clearly exposes the canonical pairs of the theory [7]. Let us see these features in the present context. The full constraint sector has to be considered. Apart from the second class constraints and the Gauss constraint, the additional constraint is given by,

$$
A_{3}^{a} \approx 0
$$

The matrix of the Poisson brackets among the constraints $\Omega_{i}^{a}$ is now given by,

$$
\left\{\Omega_{i}^{a}(x), \Omega_{j}^{b}(y)\right\}=\mathbf{C}^{a b}=\left(\begin{array}{cccc}
0 & \delta_{a b} \partial_{3} & 0 & 0 \\
\delta_{a b} \partial_{3} & 0 & \delta_{a b} \frac{\partial_{1} \partial_{3}}{\nabla^{2}} & \delta_{a b} \frac{\partial_{2} \partial_{3}}{\nabla^{2}} \\
0 & -\delta_{a b} \frac{\partial_{1} \partial_{3}}{\nabla^{2}} & 0 & \epsilon_{a b} \partial_{3} \\
0 & -\delta_{a b} \frac{\partial_{2} \partial_{3}}{\nabla^{2}} & -\epsilon_{a b} \partial_{3} & 0
\end{array}\right) \times \delta(x-y)
$$

where the first two constraints $(i=1,2)$ refer to the Gauss law and the axial gauge, respectively, while the last two $(i=3,4)$ are the second class set. The inverse matrix is given by,

$$
\mathbf{C}^{-1}=\left(\begin{array}{cccc}
0 & \delta_{b c} \frac{1}{\partial_{3}} & \epsilon_{b c} \frac{\partial_{2}}{\nabla^{2} \partial_{3}} & -\epsilon_{b c} \frac{\partial_{1}}{\nabla^{2} \partial_{3}} \\
\delta_{b c} \frac{1}{\partial_{3}} & 0 & 0 & 0 \\
\epsilon_{b c} \frac{\partial_{2}}{\nabla^{2} \partial_{3}} & 0 & 0 & \frac{1}{\partial_{3}} \epsilon_{b c} \\
-\epsilon_{b c} \frac{\partial_{1}}{\nabla^{2} \partial_{3}} & 0 & -\frac{1}{\partial_{3}} \epsilon_{b c} & 0
\end{array}\right) \times \delta(y-z)
$$

The Dirac brackets in the axial gauge are now easily found,

$$
\begin{gathered}
\left\{A_{1}^{a}(x), A_{2}^{b}(y)\right\}^{*}=\left\{A_{1}^{a}(x), A_{2}^{b}(y)\right\}-\int\left\{A_{1}^{a}(x), \Omega^{c}{ }_{i}(z)\right\} \mathbf{C}_{i j}^{c d}{ }^{-1}\left\{\Omega^{d}{ }_{j}(w), A_{2}^{b}(y)\right\} \\
=\epsilon_{a b} \frac{1}{\partial_{3}} \delta(x-y)
\end{gathered}
$$


Two observations are now in order. First, the brackets among the transverse variables,

$$
\left\{A_{T, 1}^{a}(x), A_{T, 2}^{b}(y)\right\}^{*}=\epsilon_{a b} \frac{\partial_{3}}{\nabla^{2}} \delta(x-y)
$$

reproduce the Coulomb gauge result (9). This acts as a consistency check since the above algebra must be gauge independent. Secondly, it is simple to identify the canonical pairs as $A_{1}, \pi^{1}$ and $A_{2}, \pi^{2}$ since, using the definition for the momenta and (23), it follows that,

$$
\left\{A_{1}^{b}(x), \pi_{c}^{1}(y)\right\}^{*}=\left\{A_{2}^{b}(x), \pi_{c}^{2}(y)\right\}^{*}=\frac{1}{2} \delta_{b c} \delta(x-y)
$$

The analogous situation in usual Maxwell theory may be recalled where these set of variables (without the internal indices) characterise the canonical pairs [ๆ].

\section{Symplectic Analysis}

The duality symmetric Maxwell theory is now analysed in the context of the symplectic formalism [8]. Compared to the Dirac approach this is basically a Lagrangean approach. Moreover it does not require the classification of constraints which is essential to the Dirac procedure. Details of this procedure in the context of both unconstrained and constrained systems have been provided elsewhere [9, 10]. The essential idea is to obtain the symplectic two form, the inverse of which yields the brackets of the theory. For an unconstrained system this is reasonably straightforward since the symplectic matrix is invertible. For constrained systems a possible approach [10] is to find the zero modes of the symplectic matrix which is no longer invertible. If the zero modes do not produce any restriction on the dynamical variables, it indicates the occurrence of a gauge symmetry. The natural way to proceed then is to fix a gauge. In the present model, however, explicit gauge fixing is not necessary. The brackets of the theory are directly obtained by working with the independent variables of the system. Since the symplectic analysis is suited for first order systems, the Lagrangean (4) is ideal for this purpose. Indeed, contrary to the usual Maxwell Lagrangean, conversion to the first order form by introducing auxiliary variables becomes redundant. 
To quickly recapitulate the basic tenets of the symplectic approch, [3 the geometric structure is induced by the closed symplectic two form,

$$
f^{(0)}=\frac{1}{2} f_{i j}^{(0)} d \omega_{i}^{(0)} d \omega_{j}^{(0)}
$$

where,

$$
f_{i j}^{(0)}=\frac{\partial a_{j}^{(0)}}{\partial \omega_{i}^{(0)}}-\frac{\partial a_{i}^{(0)}}{\partial \omega_{j}^{(0)}}
$$

and $a^{(0)}\left(\omega^{(0)}\right)=a_{i}^{(0)}\left(\omega^{(0)}\right) d \omega_{i}^{(0)}$ is the canonical one form defined from the original Lagrangean,

$$
L^{(0)} d t=a^{(0)}\left(\omega^{(0)}\right)-V^{(0)}\left(\omega^{(0)}\right) d t
$$

The superscript 0 implies that the original Lagrangean is being considered. In fact it is indicative of the iterative nature of the computations. Additional restrictions coming from the constraints are imposed through Lagrange mutipliers in which case one has to extend the configuration space. The corresponding Lagrangean gets modified and accordingly the superscript also changes. The process terminates once the symplectic matrix becomes invertible.

It is easy to see that the two form following from (4),

$$
f_{i j}^{a b}=-\epsilon_{a b} \epsilon_{i j k} \partial_{k} \delta(x-y)
$$

does not have an inverse. It is possible to generically denote the zero modes by,

$$
\nu_{i j}^{a b}=\partial_{i} \phi_{j}^{a b}
$$

To check whether new conditions are generated by these modes, one has to study the relation 10 ,

$$
\int \nu \frac{\partial V}{\partial \omega}=0
$$

Using (30) it is seen that the condition (31) is trivially satisfied. Hence there are no further restrictions and the system is a gauge theory. It is also clear that (4) is expressed solely in terms of the transverse components of

\footnotetext{
${ }^{3}$ The general development is given for point mechanics. The extension to field theory is self evident.
} 
the potentials so that the longitudinal components can be set equal to zero. This is incorporated by modifying the Lagrangean as,

$$
\mathcal{L}=\frac{1}{2} \epsilon^{j k i} \partial_{j} A_{k}^{a} \epsilon_{a b} \partial_{0} A_{i}^{b}-\frac{1}{4} F^{a, j k} F_{j k}^{a}+\dot{\lambda}^{a} \partial_{i} A_{i}^{a}
$$

Note that the Lagrange multiplier enforcing the transversality has been introduced by means of a time derivative to simplify the ensuing algebra. Physically this means that the transversality condition is time independent. It should perhaps be mentioned that the transversality condition enforced here is not the same as implementing the Coulomb gauge in usual Maxwell theory. In the latter case the longitudinal components are forced to vanish by putting an external gauge condition. Here, on the contrary, the duality invariant Lagrangean is already expressed in terms of the transverse components and the transversality condition separates out the independent components. This will become more clear when we discuss the same theory without introducing any Lagrange multiplier. The symplectic variables are now $A_{i}, \lambda$. The first iterated symplectic matrix is given by,

$$
f_{i j}^{a b}=\left(\begin{array}{cc}
\epsilon_{a b} \epsilon_{i j k} \partial_{k} & -\delta_{a b} \partial_{i} \\
-\delta_{a b} \partial_{j} & 0
\end{array}\right) \delta(x-y)
$$

The above matrix is nonsingular and its inverse is given by,

$$
f_{j l}^{a b(-1)}=\left(\begin{array}{cc}
\epsilon_{a b} \epsilon_{j l m} \frac{\partial_{m}}{\nabla^{2}} & -\delta_{a b} \frac{\partial_{j}}{\nabla^{2}} \\
-\delta_{a b} \frac{\partial_{l}}{\nabla^{2}} & 0
\end{array}\right) \delta(x-y)
$$

The brackets among the basic variables are now easily read-off from the first entry,

$$
\left\{A_{i}^{a}(x), A^{b}{ }_{j}(y)\right\}=\epsilon_{a b} \epsilon_{i j k} \frac{\partial_{k}}{\nabla^{\mathbf{2}}} \delta(x-y)
$$

which reproduces the Dirac algebra (19) and hence (9) also. The other brackets (10) and (11) found in the Dirac approach are nonexistant in the symplectic formalism since canonical momenta are never introduced.

The above algebra can also be obtained without 9enlarging the configuration space. This is achieved by directly working with the independent transverse variables which are isolated by choosing the following polarisation,

$$
A_{i, T}^{a}=\left(\delta_{i \alpha}-\delta_{i 3} \frac{\partial_{\alpha}}{\partial_{3}}\right) a_{\alpha}^{a} ; \quad \alpha=1,2
$$


Note that $a_{1}$ and $a_{2}$ are the two independent variables. Expressed in terms of these variables, the kinetic part of the Lagrangean (4) takes the form,

$$
\mathcal{L}_{K E}=\frac{1}{2} \epsilon_{\alpha \beta} \epsilon_{a b}\left(\partial_{3} a_{\beta}^{a}+2 \frac{\partial_{\beta} \partial_{\sigma}}{\partial_{3}} a_{\sigma}^{a}\right) \partial_{0} a_{\alpha}^{b}
$$

Thus proceeding as before, we get the symplectic matrix as,

$$
f_{\alpha \beta}^{a b}=\left(\begin{array}{cc}
0 & \epsilon_{a b} \frac{\nabla^{2}}{\partial_{3}} \\
-\epsilon_{a b} \frac{\nabla^{2}}{\partial_{3}} & 0
\end{array}\right) \delta(x-y)
$$

Its inverse is given by,

$$
f^{-1}=\left(\begin{array}{cc}
0 & \epsilon_{a b} \frac{\partial_{3}}{\nabla^{2}} \\
-\epsilon_{a b} \frac{\partial_{3}}{\nabla^{2}} & 0
\end{array}\right) \times \delta(x-y)
$$

The only nontrivial bracket is given by the off-diagonal entry,

$$
\left\{a_{1}^{a}(x), a_{2}^{b}(y)\right\}=\epsilon_{a b} \frac{\partial_{3}}{\nabla^{2}} \delta(x-y)
$$

It is easy to verify that this is the same algebra found earlier in (9) or (35).

Before concluding this section we discuss the axial gauge formulation. This gauge is imposed as,

$$
\mathcal{L}^{(1)}=\frac{1}{2} \epsilon^{j k i} \partial_{j} A_{k}{ }^{a} \epsilon_{a b} \partial_{0} A_{i}^{b}-\frac{1}{4} F^{a, j k} F^{a}{ }_{j k}+\dot{\lambda}^{(a)} A_{3}^{a}
$$

The above first iterated Lagrangean leads to a nonsingular symplectic matrix. Inverting it the relevant brackets are obtained. These correctly reproduce (23).

\section{Conclusions}

The present paper has shown that there are several facets to the constrained dynamics of duality symmetric Maxwell theory. An intriguing feature was the appearance of a reducible set of second class constraints together with first class constraints, whereas the normal Maxwell Lagrangean has only the latter. Nevertheless by suitably accounting for the reducibility using an 
enlargement prescription, Dirac brackets among the physical variables were directly obtained. In other words any explicit reference to either the first class constraints or the gauge fixing, which has been the standard approach [5] using the Coulomb gauge, was avoided. This is also a distinct point of departure from the conventional constrained analysis in the Maxwell theory where the full set of constraints is required to calculate the Dirac brackets. For the axial gauge, on the other hand, it was necessary to take the complete set of constraints simultaneously. In this case a close parallel with the usual Maxwell analysis was established since similar canonical pairs, modified by proper internal indices, were obtained.

The Dirac anlysis was followed by a symplectic approach. Indeed the first order nature of the duality symmetric Lagrangean naturally lends itself to such an analysis. It is not surprising therefore that the symplectic formalism yields results in a more neat and compact fashion compared to the Dirac procedure. This may be contrasted with the normal second order Maxwell Lagrangean where the application of either the Dirac or symplectic formulations is more a matter of taste. The results in the symplectic approach were obtained both in the gauge independent and gauge fixed versions and were shown to agree with the Dirac analysis. As is characteristic of the symplectic formalism, no reference was made to the nature of the constraints. In fact the natural first order form of the Lagrangean simplified the problem considerably so that no constraints emerged from the analysis. The gauge symmetry was shown to be a direct consequence of the redundancy in the degrees of freedom.

A possible generalisation can be made for other duality symmetric models. Since these are first order Lagrangeans, it is obvious that the symplectic formalism would be ideal for discussing the constrained structure for such models. It also seems reasonable to presume that this constrained structure would be quite distinctive from that of the respective parent Lagrangeans. This opens up the possibility of obtaining new features from the duality symmetric Lagrangeans and gives us an added reason to study such systems which are quite independent of confining ones attention to just probe the nature of duality symmetry. 


\section{References}

[1] D.I. Olive, Nucl. Phys. B(Proc. Suppl.) 58 (1997) 43

[2] S. Deser and C. Teitelboim, Phys. Rev. D13 (1976) 1572.

[3] J. Schwarz and A. Sen, Nucl. Phys. B411 (1994) 35.

[4] R. Banerjee and C. Wotzasek, hep-th/9805109; To appear in Nucl. Phys.B.

[5] H.O.Girotti ,Phy.Rev. D55 (1997) 5136; I. Martin and A. Restuccia, Phys. Lett. B323 (1994) 311.

[6] R. Banerjee and J. Barcelos-Neto , Ann Phys. 265 (1998) 134.

[7] A. Hanson, T. Regge and C. Teitelboim, Constrained hamiltonian systems; (Acad. Naz. dei Lincei, Rome, 1976).

[8] V.I. Arnold, Mathematical methods of classical mechanics, Springer 1978.

[9] L. Faddeev and R. Jackiw, Phys. Rev. Lett. 60 (1988) 1692.

[10] J. Barcelos-Neto and C. Wotzasek Mod. Phys. Lett. A7 (1992) 1737. 